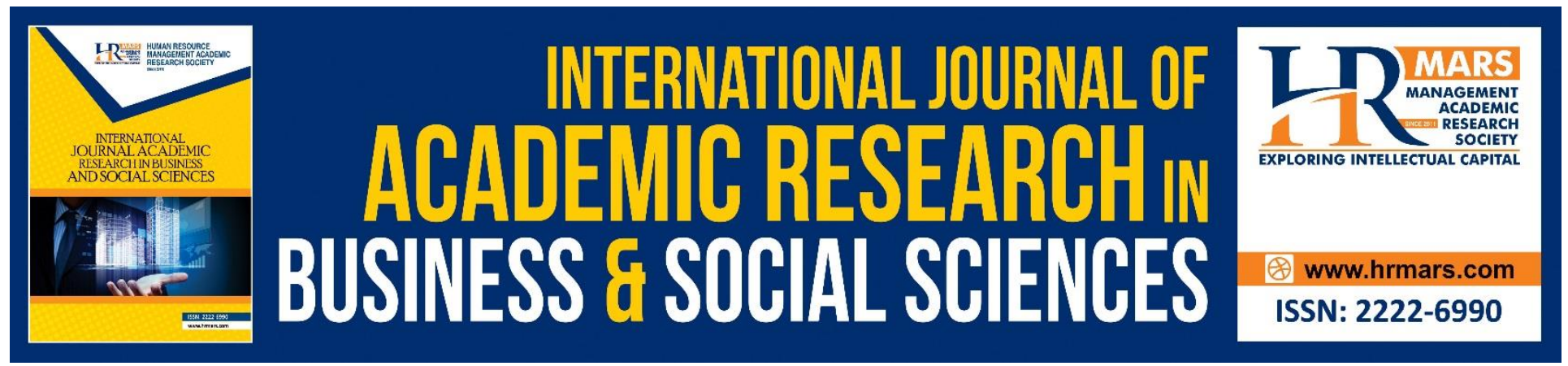

\title{
Entrepreneurship Education and Self-Employment in Nigeria: Evaluation of Perceived Barriers to Actualization
}

Yusuff, Mulkat Ajibola

To Link this Article: http://dx.doi.org/10.6007/IJARBSS/v8-i12/5417

DOI: $10.6007 /$ IJARBSS/v8-i12/5417

Received: 07 Dec 2018, Revised: 29 Dec 2018, Accepted: 07 Jan 2019

Published Online: 09 Jan 2019

In-Text Citation: (Yusuff, 2018)

To Cite this Article: Yusuff, M. A. (2018). Entrepreneurship Education and Self-Employment in Nigeria: Evaluation of Perceived Barriers to Actualization. International Journal of Academic Research in Business and Social Sciences, 8(12), 2160-2174.

Copyright: (C) 2018 The Author(s)

Published by Human Resource Management Academic Research Society (www.hrmars.com)

This article is published under the Creative Commons Attribution (CC BY 4.0) license. Anyone may reproduce, distribute, translate and create derivative works of this article (for both commercial and non-commercial purposes), subject to full attribution to the original publication and authors. The full terms of this license may be seen

at: http://creativecommons.org/licences/by/4.0/legalcode

\section{Vol. 8, No. 12, 2018, Pg. $2160-2174$}

Full Terms \& Conditions of access and use can be found at http://hrmars.com/index.php/pages/detail/publication-ethics 


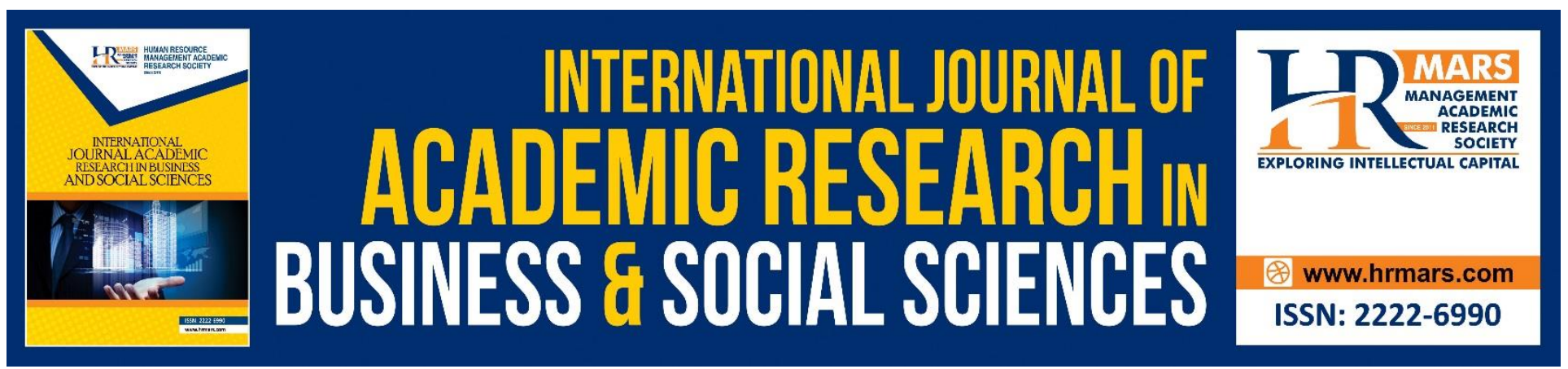

\title{
Entrepreneurship Education and Self-Employment in Nigeria: Evaluation of Perceived Barriers to Actualization
}

\author{
Yusuff, Mulkat Ajibola \\ The Federal Polytechnic, Ilaro, Ogun State, Nigeria \\ Email:mulkat.ajibola@gmail.com
}

\begin{abstract}
Following the integration of Entrepreneurship Education into the curricular of all tertiary institutions in Nigeria, much light has been shed on the influence of entrepreneurship education on the probability of graduates being self-employed after graduation. However, there is a lack of systemic investigation in the area of barriers to entrepreneurship and how they affect the intention of becoming entrepreneur. Consequently, the aim of this study is to identify perceived barriers which prevent graduates from self-employment. Using responses from 200 graduates of tertiary institutions on a 5 point Likert Scale, data is analysed with Descriptive statistics, Correlation and Component Factor Analysis having subjected the validity check on questionnaires' responses to Cronbach Alpha test. The findings showed that difficulty involved in securing loan from financial institutions, fear of risk, inability to generate new business idea, weak economic environment and difficulty in locating customer were major obstacles to starting a business with cumulative variance of $70.15 \%$. These effects are found to be positive on barriers to graduates' decision to be self-employed with respective eigenvalues of 4.030, $2.674,2.216,1.194$ and 1.108 . The study therefore suggests that the policy maker should implement sound economic policies to boost the country's economic environment, simplify the bureaucracy of assessing loan and increase the campaign to re-orientate Nigerians towards Nigerian products. The institutions of learning also need to do more in the area of mentoring to alley the fear of risk and adopt interactive and practical operational methods of teaching in delivering entrepreneurship education.
\end{abstract}

Keywords: Actualization, Barriers, Graduates, Entrepreneurship Education, Self-Employment.

\section{INTRODUCTION}

The empirical link between entrepreneurship education and entrepreneurial intention have received ample attention from researchers across the world (Harrison and Hart, 1989; Fleming, 
1992; Owusu-Ansah and Poku, 2012) and Nigeria in particular (Ekpoh and Edet, 2011; Nnadozie, Akanwa, Nnadozie 2013; Onuma, 2016). These studies have found statistical significant relationship between entrepreneurship education and entrepreneurial intention.

Entrepreneurial intention is one's will to get involved in entrepreneurial activity and is considered as potential predictor of entrepreneurial start up. Dohse and Walter (2010) defined entrepreneurship intention as the willingness of an individual to perform entrepreneurial cbehaviour, to engage in entrepreneurial action, to be self-employed or to establish new business. According to Kolvereid and Isaksen (2006). Intention to become self-employed determine actual entry into self-employment.

However, the constraints and barriers to entrepreneurship act against one's intention, making realisation of the intentions into entrepreneurial action difficult or even impossible. In the words of Carayannis, Evans and Hanson (2003) including Pittaway and Cole (2007), the intention of an individual to become entrepreneur is shaped by his perception of barriers to business start-up, cultural values and the environment in which he is located. In the same vein, Luthje and Franke (2003) as mentioned in lakovleva (2014) posit that entrepreneurial intentions relate to perceived barriers to business creation, cultural values and the environments' infrastructure established to support entrepreneurs.

When constraints and barriers exist, the engagement into entrepreneurial activity become difficulty and even practically impossible. There is need to empirically investigate barriers perceived by graduates of Nigerian institutions of higher learning to their entrepreneurial intention. Identifying the perceived barriers will provide means of dramatically improve entrepreneurship education and remind the government of the need to give priority to the necessary support for business start-up.

\section{Conceptual Review}

\section{Entrepreneurship Education}

The concept of entrepreneurship education is nebulous and many academic researchers have contributed their perspectives on the concept of entrepreneurship education. Towobola and Raimi (2011) conceptualize entrepreneurship education as pragmatic and meaningful interaction between learner and instructor for the purpose of developing in the learner the ability to identify, evaluate and generate ideas and solving problems in a unique way. Entrepreneurship education stimulate consciousness towards self-employment. This is because it is a programme designed to prepare students with the required skills and knowledge to be able to identify opportunities, understand a customer's perception, generate new ideas, and developing business plans as well as understanding and evaluating environmental, institutional and political issues (Cheng, Chan \& Mahmood, 2009 as mentioned in Rosni, Md Aminul \& Jocelyne, 2015).

To Mauchi et.al (2011), entrepreneurship education is a process of providing individuals with ability to recognize commercial opportunities and the knowledge, skills and attitudes to act on them. Okiti (2009 as mentioned in Akpan and Etor 2013) explained entrepreneurship education 
as the gateway to job opportunities and job creation which would constantly enhance selfreliance and self-employment among graduates. Adebayo and Kolawole (2013) also described entrepreneurship education as specializes training given the students or trainees to acquire skills, ideas, managerial abilities and capabilities for self-employment than employed for wage pay. Entrepreneurship education as an area of study has those attitudes and skills essential for responding to one's environment in the process of conceiving, starting and managing a business enterprise.

From the fore going, entrepreneurship education is generally view as a type of training designed to change the orientation and attitude of the recipients (students) and in the process equip with skills and knowledge to enable them start and manage business enterprise.

\section{Self-employment}

Self-employment refers to a situation in which an individual conceives, creates, manages and assumes responsibility for a business enterprise rather than working for others in a paid employment. According to Citizen Information (2014), a person is self-employed when he/she owns a business rather than working for an employer. Abdul Karim (2012) also describes selfemployment as an act of working for oneself. Self-employment avail the opportunity of generating one's income directly from customers, clients or other organizations as opposed to earning salary as an employee.

\section{Barriers to Entrepreneurship}

Barriers to entrepreneurship relate to "precipitating events", moderating the link between entrepreneurial intention and actual efforts to start an enterprise (Luthje \& Franke, 2003). They are factors impeding entrepreneurship intentions and are also constraints to one's engagement to self-employment. Sitaridis and Kitsios (2016) after extensive study of literature classified barriers to entrepreneurship into internal barriers and external barriers. Internal barriers are fear of risk, lack of confidence, insufficient entrepreneurial skill and negative attitude towards entrepreneurship. External barriers include poor access to capital, lack of institutional support, resource constraints (human and infrastructure), market related barriers (tough competition, difficulty to find customers), time consuming registration procedures, bureaucracy, administrative burden and political future uncertainty among others. Whichever ways these barriers are classified; they are important factors capable of discouraging the start-up of new businesses.

\section{Empirical Studies}

Scholars have empirically provided insight into perceived barriers to entrepreneurship and their relative importance in impeding actualization of entrepreneurship intention. Tanveer et al (n.d) investigate barriers and hurdles to become entrepreneur among 114 undergraduates and post graduates in Pakistan. Their findings revealed that lack of skills, lack of assistance, lack of capital, lack of awareness and fear of future are major hurdles to starting a business. The work Sitaridis and Kitsios (2016) showed that financial and market constraints have significant negative effect on the entrepreneurial intentions and motives of students towards entrepreneurship in Greece. 
Van der Zwan, Zuurhout \& Hessels (2013) assessed possible mediating role of perceived financial impediments, perceived administrative complexities and fear of business failure on entrepreneurial intentions of 20,000 individuals in 36 European countries, their result revealed perceived administrative complexities hinders individual most to become self-employed. Smith and Beasley (2011) in their study of factors that influence seven graduate students in the creative and digital industries to start and run their own businesses in the United Kingdom identified lack of financial resources, lack of general business knowledge, contradictory advisory support from external agencies and lack of sector-specific mentors as impeding factors. In a survey of 226 Malaysian postgraduate students Sandhu, Sidique and Riaz (2010) confirmed that most significant barriers to entrepreneurial intentions are lack of social networking followed by the lack of resources and aversion to risk.

From the fore going it is shown that the types of barriers to entrepreneurship varies across countries. This study therefore set to empirically identify and specify those factors which Nigerian graduates that had received entrepreneurship education perceived as hindrance to set up their own businesses.

\section{Methodology}

Survey method was adopted in carrying out this study because of the large population of Nigerian graduates. This is line with Babbie's (2004) submission as mentioned in Nnadozie et al (2013) that the survey method allows researchers to draw a large sample that is representative of the total population. The data used were collected through structured questionnaire containing 16 statements on barriers to entrepreneurship as obtained in literature. Each statement is measured on 5-point Likert scale ranging from strongly disagree to strongly agree. (1. Strongly disagree, 2. Disagree, 3. Undecided, 4. Agree, 5. Strongly agree). The respondents were graduates who has positive entrepreneurial inclination and on National Youth Service Corps (NYSC) in Yewa South local government, Ogun State, Nigeria. They were carefully selected through random sampling method. The reliability of the data was measured with Cronbach's alpha. Descriptive Statistics and intercorrelaton analysis were applied to scale statements to examine the order of importance. Principal component analysis which is a statistical procedure that extracts important variables (by removal of redundant variables) in a data set was also employed as data reduction technique to ascertain whether or not there exist a set of stable and reliable factors germane to the study.

\section{Results and Discussion}

Reliability analysis made returned a Cronbach's Alpha statistic of 0.764 in table 1 , which indicates a high level of internal consistency for the 5-point likert scales used under study. This also shows that the scales were found to be reliable for the research work thereby showing individual contribution of all the barrier factors for decision making process.

Table 1: $\quad$ Reliability Statistics

\begin{tabular}{|r|r|r|r|}
\hline Cronbach's Alpha & Cronbach's Alpha Based on Standardized Items & N of Items \\
\hline .764 & .765 & 16 \\
\hline
\end{tabular}


Figure 1 to 3 showed demographic features of the respondents. Majority of the respondents (69\%) were male graduates; $57 \%$ were degree holders; with majority (62\%) between age brackets 21-25 years respectively. Analysis of age and educational qualification of the respondents serves as a factor for the consistency and reliability of the research questions based on their level of maturity and educational background.
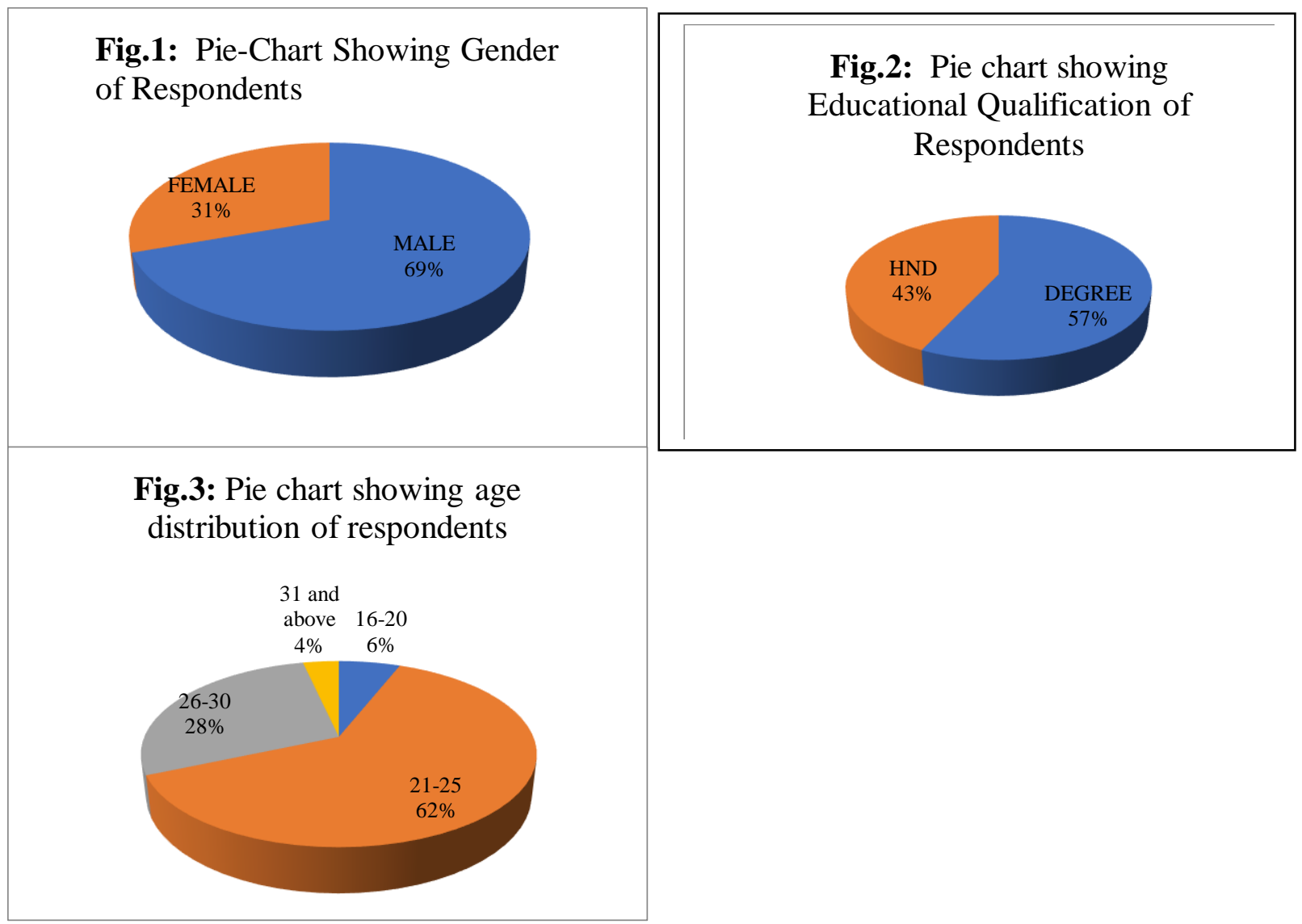

The descriptive statistics in table 2 revealed that an me varrers isseu were importani in mniting the respondents' decision to be self-employed. The average score for all the tests have a similar spread, with fear of risk being the most significant inhibiting factor followed by difficulty in obtaining bank loan. 
INTERNATIONAL JOURNAL OF ACADEMIC RESEARCH IN BUSINESS AND SOCIAL SCIENCES

Vol. 8, No. 12, Dec, 2018, E-ISSN: $2222-6990$ (C) 2018 HRMARS

Table 2: $\quad$ Descriptive Statistics

\begin{tabular}{|l|r|r|r|}
\hline & Mean & \multicolumn{1}{|c|}{$\begin{array}{c}\text { Std. } \\
\text { Deviation }\end{array}$} & \multicolumn{1}{|c|}{ Nnalysis } \\
\hline Fear of Risk & 3.92 & 1.376 & 200 \\
Fear of failure & 3.74 & 1.304 & 200 \\
Inability to develop new business idea & 2.71 & 1.147 & 200 \\
Inability to write business plan & 2.7250 & 1.36333 & 200 \\
Insufficient practical skill to start business & 3.17 & 1.259 & 200 \\
Lack of savings & 3.70 & 1.233 & 200 \\
Difficulty in obtainig bank finance & 3.82 & 1.287 & 200 \\
Complicated legal and administrative procedure for business & 3.13 & 1.223 & 200 \\
registration. & 3.17 & 1.269 & 200 \\
Poor implementation of import related legislation & 3.78 & 1.066 & 200 \\
Weak economic environment & 3.63 & 1.100 & 200 \\
Fear of uncertainty & 3.50 & 1.375 & 200 \\
Low demand for Nigerian made goods/services. & 2.76 & 1.401 & 200 \\
Difficult to find customers & 3.69 & 1.340 & 200 \\
Tough Competition & 3.37 & 1.436 & 200 \\
Poor state of infrastructure in the country & 2.25 & 1.197 & 200 \\
Lack of family support & &
\end{tabular}

The starting point for all factor analysis techniques is the correlation matrix. All factor analysis (Principal Components) techniques try to clump subgroups of variables together based upon their correlations and often one can get a feel for what the factors are going to be by examining the correlation matrix and spotting clusters of high correlations between groups of variables. The result of correlation matrix in table 3 indicates that there exists positive degree of relationship between majorities of the barrier factors considered. According to Norman and Streiner (2001), if there are few correlations above 0.3 it is a waste of time of carrying on with the analysis. Table 3 further revealed that majority of the factors have estimated correlations above 0.3 coefficients. Clearly we do not have that problem. 


\begin{tabular}{|c|c|c|c|c|c|c|c|c|c|c|c|c|c|c|c|c|c|}
\hline \multicolumn{18}{|c|}{ Table 3: Correlations } \\
\hline & & 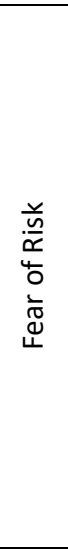 & 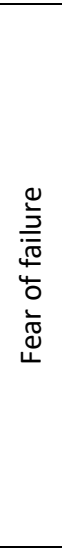 & 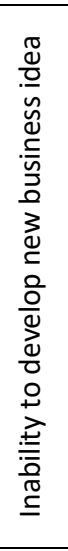 & 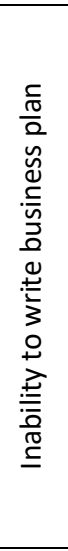 & 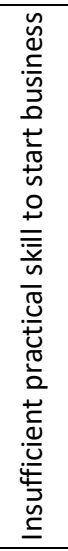 & 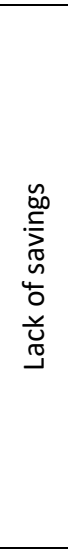 & 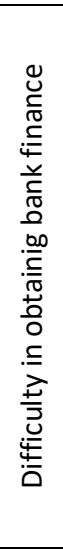 & 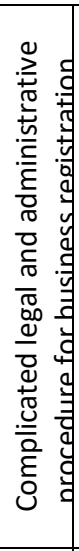 & 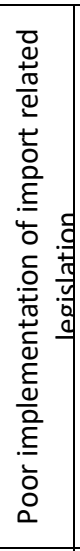 & 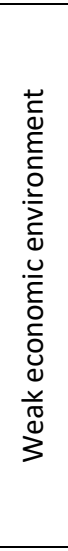 & 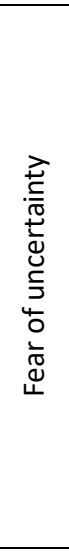 & 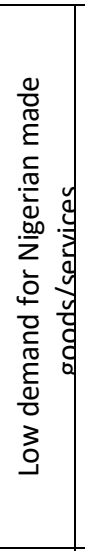 & 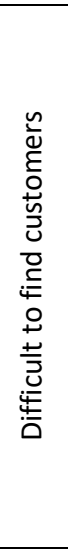 & 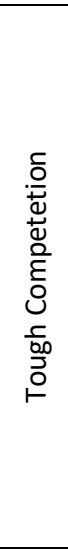 & 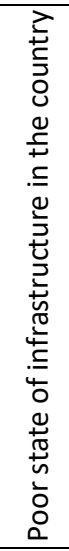 & 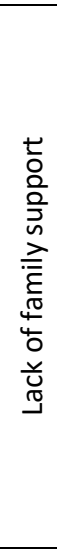 \\
\hline \multirow{6}{*}{$\begin{array}{l}\text { Fear of } \\
\text { Risk }\end{array}$} & Pearson & 1 & .56 & .11 & - & - & - & - & - & - & .08 & .31 & - & - & .25 & - & .05 \\
\hline & Correlati & & 8 & 9 & .02 & .14 & .05 & .24 & .20 & .43 & 7 & 2 & .05 & .02 & 9 & .09 & 5 \\
\hline & & & & & 3 & 9 & 6 & 4 & 0 & 8 & & & 9 & 6 & & 7 & \\
\hline & Sig. (2- & & .00 & .09 & .75 & .03 & .43 & .00 & .00 & .00 & .21 & .00 & .40 & .71 & .00 & .17 & .44 \\
\hline & tailed) & & 0 & 4 & 2 & 5 & 1 & 1 & 5 & 0 & 9 & 0 & 9 & 8 & 0 & 2 & 2 \\
\hline & $\mathrm{N}$ & 200 & 200 & 200 & 200 & 200 & 200 & 200 & 200 & 200 & 200 & 200 & 200 & 200 & 200 & 200 & 20 \\
\hline \multirow{6}{*}{$\begin{array}{l}\text { Fear of } \\
\text { failure }\end{array}$} & Pearson & .56 & 1 & .37 & .09 & .22 & .25 & .14 & - & .11 & .10 & .24 & .35 & - & .43 & .35 & .22 \\
\hline & $\begin{array}{l}\text { Correlati } \\
\text { on }\end{array}$ & 8 & & 2 & 8 & 2 & 0 & 9 & $\begin{array}{r}.00 \\
8\end{array}$ & 2 & 3 & 0 & 8 & $\begin{array}{r}.00 \\
4\end{array}$ & 0 & 7 & 5 \\
\hline & Sig. (2- & .00 & & .00 & .16 & .00 & .00 & .03 & .91 & .11 & .14 & .00 & .00 & .95 & .00 & .00 & .00 \\
\hline & tailed) & 0 & & 0 & 7 & 2 & 0 & 6 & 2 & 5 & 6 & 1 & 0 & 4 & 0 & 0 & 1 \\
\hline & $\mathrm{N}$ & 200 & 200 & 200 & 200 & 200 & 200 & 200 & 200 & 200 & 200 & 200 & 200 & 200 & 200 & 200 & 20 \\
\hline & & & & & & & & & & & & & & & & & 0 \\
\hline \multirow{5}{*}{$\begin{array}{l}\text { Inability } \\
\text { to } \\
\text { develop } \\
\text { new } \\
\text { business } \\
\text { idea }\end{array}$} & Pearson & .11 & .37 & 1 & .78 & .27 & - & - & .20 & .41 & .05 & .06 & .28 & .38 & .19 & .16 & .45 \\
\hline & $\begin{array}{l}\text { Correlati } \\
\text { on }\end{array}$ & 9 & 2 & & 7 & 1 & $\begin{array}{r}.11 \\
7 \\
\end{array}$ & $\begin{array}{r}.14 \\
9 \\
\end{array}$ & 2 & 1 & 8 & 3 & 1 & 7 & 1 & 3 & 6 \\
\hline & Sig. (2- & .09 & .00 & & .00 & .00 & .09 & .03 & .00 & .00 & .41 & .37 & .00 & .00 & .00 & .02 & .00 \\
\hline & tailed) & 4 & 0 & & 0 & 0 & 8 & 6 & 4 & 0 & 8 & 4 & 0 & 0 & 7 & 1 & 0 \\
\hline & $\mathrm{N}$ & 200 & 200 & 200 & 200 & 200 & 200 & 200 & 200 & 200 & 200 & 200 & 200 & 200 & 200 & 200 & 20 \\
\hline \multirow{6}{*}{$\begin{array}{l}\text { Inability } \\
\text { to write } \\
\text { business } \\
\text { plan }\end{array}$} & Pearson & - & .09 & .78 & 1 & .21 & - & - & .21 & .34 & .03 & .05 & .18 & .36 & .04 & .00 & .43 \\
\hline & Correlati & .02 & 8 & 7 & & 4 & .21 & .20 & 7 & 7 & 8 & 2 & 8 & 3 & 0 & 5 & 3 \\
\hline & & 3 & & & & & 2 & 6 & & & & & & & & & \\
\hline & Sig. (2- & .75 & .16 & .00 & & .00 & .00 & .00 & .00 & .00 & .59 & .46 & .00 & .00 & .57 & .94 & .00 \\
\hline & tailed) & 2 & 7 & 0 & & 2 & 3 & 3 & 2 & 0 & 6 & 9 & 8 & 0 & 0 & 0 & 0 \\
\hline & $\mathrm{N}$ & 200 & 200 & 200 & 200 & 200 & 200 & 200 & 200 & 200 & 200 & 200 & 200 & 200 & 200 & 200 & 20 \\
\hline \multirow{6}{*}{$\begin{array}{l}\text { Insufficie } \\
\text { nt } \\
\text { practical } \\
\text { skill to } \\
\text { start } \\
\text { business }\end{array}$} & Pearson & - & .22 & .27 & .21 & 1 & .27 & 19 & .24 & .36 & .23 & .13 & .29 & .04 & .14 & .26 & - \\
\hline & Correlati & .14 & 2 & 1 & 4 & & 2 & 8 & 4 & 3 & 3 & 9 & 5 & 5 & 7 & 7 & .07 \\
\hline & & 9 & & & & & & & & & & & & & & & 4 \\
\hline & Sig. (2- & .03 & .00 & .00 & .00 & & .00 & .00 & .00 & .00 & .00 & .04 & .00 & .52 & .03 & .00 & .30 \\
\hline & tailed) & 5 & 2 & 0 & 2 & & 0 & 5 & 0 & 0 & 1 & 9 & 0 & 4 & 8 & 0 & 0 \\
\hline & $\mathrm{N}$ & 200 & 200 & 200 & 200 & 200 & 200 & 200 & 200 & 200 & 200 & 200 & 200 & 200 & 200 & 200 & 20 \\
\hline \multirow{5}{*}{$\begin{array}{l}\text { Lack of } \\
\text { savings }\end{array}$} & Pearson & - & .25 & - & - & .27 & 1 & .68 & .27 & .25 & .19 & .23 & .30 & - & .12 & .38 & - \\
\hline & Correlati & .05 & 0 & .11 & .21 & 2 & & 4 & 5 & 2 & 0 & 8 & 0 & .15 & 7 & 4 & .06 \\
\hline & & 6 & & 7 & 2 & & & & & & & & & 0 & & & 5 \\
\hline & Sig. (2- & .43 & .00 & .09 & .00 & .00 & & .00 & .00 & .00 & .00 & .00 & .00 & .03 & .07 & .00 & .36 \\
\hline & tailed) & 1 & 0 & 8 & 3 & 0 & & 0 & 0 & 0 & 7 & 1 & 0 & 4 & 3 & 0 & 1 \\
\hline
\end{tabular}


INTERNATIONAL JOURNAL OF ACADEMIC RESEARCH IN BUSINESS AND SOCIAL SCIENCES

Vol. 8, No. 12, Dec, 2018, E-ISSN: 2222-6990 @ 2018 HRMARS

\begin{tabular}{|c|c|c|c|c|c|c|c|c|c|c|c|c|c|c|c|c|c|}
\hline & $\mathrm{N}$ & 200 & 200 & 200 & 200 & 200 & 200 & 200 & 200 & 200 & 200 & 200 & 200 & 200 & 200 & 200 & $\begin{array}{r}20 \\
0\end{array}$ \\
\hline \multirow{5}{*}{$\begin{array}{l}\text { Difficulty } \\
\text { in } \\
\text { obtaining } \\
\text { bank } \\
\text { finance }\end{array}$} & Pearson & - & .14 & - & - & .19 & .68 & 1 & .32 & .28 & .28 & .14 & .39 & .03 & .12 & .67 & - \\
\hline & $\begin{array}{l}\text { Correlati } \\
\text { on }\end{array}$ & $\begin{array}{r}.24 \\
4\end{array}$ & 9 & $\begin{array}{r}.14 \\
9\end{array}$ & $\begin{array}{r}.20 \\
6\end{array}$ & 8 & 4 & & 7 & 7 & 6 & 7 & 7 & 4 & 7 & 8 & $\begin{array}{r}.34 \\
6\end{array}$ \\
\hline & Sig. (2- & .00 & .03 & .03 & .00 & .00 & .00 & & .00 & .00 & .00 & .03 & .00 & .62 & .07 & .00 & .00 \\
\hline & tailed) & 1 & 6 & 6 & 3 & 5 & 0 & & 0 & 0 & 0 & 7 & 0 & 8 & 3 & 0 & 0 \\
\hline & $\mathrm{N}$ & 200 & 200 & 200 & 200 & 200 & 200 & 200 & 200 & 200 & 200 & 200 & 200 & 200 & 200 & 200 & $\begin{array}{r}20 \\
0 \\
\end{array}$ \\
\hline \multirow{5}{*}{$\begin{array}{l}\text { Complicat } \\
\text { ed legal } \\
\text { and } \\
\text { administr } \\
\text { ative } \\
\text { procedur } \\
\text { e for } \\
\text { business } \\
\text { registrati } \\
\text { on. }\end{array}$} & Pearson & - & - & .20 & .21 & .24 & .27 & .32 & 1 & .64 & .34 & .03 & .29 & .24 & - & .39 & - \\
\hline & $\begin{array}{l}\text { Correlati } \\
\text { on }\end{array}$ & $\begin{array}{r}.20 \\
0\end{array}$ & $\begin{array}{r}.00 \\
8\end{array}$ & 2 & 7 & 4 & 5 & 7 & & 4 & 1 & 1 & 2 & 6 & $\begin{array}{r}.13 \\
5\end{array}$ & 4 & $\begin{array}{r}.07 \\
3\end{array}$ \\
\hline & Sig. (2- & .00 & .91 & .00 & .00 & .00 & .00 & .00 & & .00 & .00 & .66 & .00 & .00 & .05 & .00 & .30 \\
\hline & tailed) & 5 & 2 & 4 & 2 & 0 & 0 & 0 & & 0 & 0 & 0 & 0 & 0 & 6 & 0 & 8 \\
\hline & $\mathrm{N}$ & 200 & 200 & 200 & 200 & 200 & 200 & 200 & 200 & 200 & 200 & 200 & 200 & 200 & 200 & 200 & $\begin{array}{r}20 \\
0\end{array}$ \\
\hline \multirow{5}{*}{$\begin{array}{l}\text { Poor } \\
\text { implemen } \\
\text { tation of } \\
\text { import } \\
\text { related } \\
\text { legislatio } \\
\mathrm{n}\end{array}$} & Pearson & - & .11 & .41 & .34 & .36 & .25 & .28 & .64 & 1 & .36 & - & .33 & .09 & .13 & .44 & .15 \\
\hline & $\begin{array}{l}\text { Correlati } \\
\text { on }\end{array}$ & $\begin{array}{r}.43 \\
8\end{array}$ & 2 & 1 & 7 & 3 & 2 & 7 & 4 & & 2 & $\begin{array}{r}.05 \\
1\end{array}$ & 5 & 4 & 8 & 6 & 4 \\
\hline & Sig. (2- & .00 & .11 & .00 & .00 & .00 & .00 & .00 & .00 & & .00 & .47 & .00 & .18 & .05 & .00 & .02 \\
\hline & tailed) & 0 & 5 & 0 & 0 & 0 & 0 & 0 & 0 & & 0 & 1 & 0 & 7 & 1 & 0 & 9 \\
\hline & $\mathrm{N}$ & 200 & 200 & 200 & 200 & 200 & 200 & 200 & 200 & 200 & 200 & 200 & 200 & 200 & 200 & 200 & $\begin{array}{r}20 \\
0\end{array}$ \\
\hline \multirow{5}{*}{$\begin{array}{l}\text { Weak } \\
\text { economic } \\
\text { environm } \\
\text { ent }\end{array}$} & Pearson & .08 & .10 & .05 & .03 & .23 & .19 & .28 & .34 & .36 & 1 & .35 & .11 & .02 & .27 & .41 & - \\
\hline & $\begin{array}{l}\text { Correlati } \\
\text { on }\end{array}$ & 7 & 3 & 8 & 8 & 3 & 0 & 6 & 1 & 2 & & 8 & 9 & 8 & 8 & 0 & $\begin{array}{r}.10 \\
3 \\
\end{array}$ \\
\hline & Sig. (2- & .21 & .14 & .41 & .59 & .00 & .00 & .00 & .00 & .00 & & .00 & .09 & .69 & .00 & .00 & .14 \\
\hline & tailed) & 9 & 6 & 8 & 6 & 1 & 7 & 0 & 0 & 0 & & 0 & 3 & 0 & 0 & 0 & 6 \\
\hline & $\mathrm{N}$ & 200 & 200 & 200 & 200 & 200 & 200 & 200 & 200 & 200 & 200 & 200 & 200 & 200 & 200 & 200 & 20 \\
\hline \multirow{5}{*}{$\begin{array}{l}\text { Fear of } \\
\text { uncertain } \\
\text { ty }\end{array}$} & Pearson & .31 & .24 & .06 & .05 & .13 & .23 & .14 & .03 & - & .35 & 1 & .28 & .03 & .22 & .08 & - \\
\hline & Correlati & 2 & 0 & 3 & 2 & 9 & 8 & 7 & 1 & .05 & 8 & & 6 & 9 & 3 & 1 & .02 \\
\hline & Sig. (2- & .00 & .00 & .37 & .46 & .04 & .00 & .03 & .66 & $\begin{array}{r}1 \\
47\end{array}$ & .00 & & .00 & .58 & .00 & .25 & $\frac{5}{.72}$ \\
\hline & tailed) & 0 & 1 & 4 & 9 & 9 & 1 & 7 & 0 & 1 & 0 & & 0 & 2 & 2 & 6 & 2 \\
\hline & $\mathrm{N}$ & 200 & 200 & 200 & 200 & 200 & 200 & 200 & 200 & 200 & 200 & 200 & 200 & 200 & 200 & 200 & 20 \\
\hline \multirow{5}{*}{$\begin{array}{l}\text { Low } \\
\text { demand } \\
\text { for } \\
\text { Nigerian } \\
\text { made } \\
\text { goods/ser } \\
\text { vices. }\end{array}$} & Pearson & - & .35 & .28 & .18 & .29 & .30 & .39 & .29 & .33 & .11 & .28 & 1 & .28 & .33 & .53 & .03 \\
\hline & $\begin{array}{l}\text { Correlati } \\
\text { on }\end{array}$ & $\begin{array}{r}.05 \\
9\end{array}$ & 8 & 1 & 8 & 5 & 0 & 7 & 2 & 5 & 9 & 6 & & 1 & 3 & 7 & 6 \\
\hline & Sig. (2- & .40 & .00 & .00 & .00 & .00 & .00 & .00 & .00 & .00 & .09 & .00 & & .00 & .00 & .00 & .61 \\
\hline & tailed) & 9 & 0 & 0 & 8 & 0 & 0 & 0 & 0 & 0 & 3 & 0 & & 0 & 0 & 0 & 4 \\
\hline & $\mathrm{N}$ & 200 & 200 & 200 & 200 & 200 & 200 & 200 & 200 & 200 & 200 & 200 & 200 & 200 & 200 & 200 & $\begin{array}{r}20 \\
0\end{array}$ \\
\hline \multirow{6}{*}{$\begin{array}{l}\text { Difficult } \\
\text { to find } \\
\text { customer } \\
\mathrm{s}\end{array}$} & Pearson & - & - & .38 & .36 & .04 & - & .03 & .24 & .09 & .02 & .03 & .28 & 1 & - & .19 & - \\
\hline & Correlati & .02 & .00 & 7 & 3 & 5 & .15 & 4 & 6 & 4 & 8 & 9 & 1 & & .01 & 1 & .02 \\
\hline & & 6 & 4 & & & & 0 & & & & & & & & 1 & & 5 \\
\hline & Sig. (2- & .71 & .95 & .00 & .00 & .52 & .03 & .62 & .00 & .18 & .69 & .58 & .00 & & .87 & .00 & .72 \\
\hline & tailed) & 8 & 4 & 0 & 0 & 4 & 4 & 8 & 0 & 7 & 0 & 2 & 0 & & 7 & 7 & 8 \\
\hline & $\mathrm{N}$ & 200 & 200 & 200 & 200 & 200 & 200 & 200 & 200 & 200 & 200 & 200 & 200 & 200 & 200 & 200 & $\begin{array}{r}20 \\
0\end{array}$ \\
\hline
\end{tabular}


INTERNATIONAL JOURNAL OF ACADEMIC RESEARCH IN BUSINESS AND SOCIAL SCIENCES Vol. 8, No. 12, Dec, 2018, E-ISSN: 2222-6990 @ 2018 HRMARS

\begin{tabular}{|c|c|c|c|c|c|c|c|c|c|c|c|c|c|c|c|c|c|}
\hline \multirow{3}{*}{$\begin{array}{l}\text { Tough } \\
\text { Competet } \\
\text { ion }\end{array}$} & $\begin{array}{l}\text { Pearson } \\
\text { Correlati } \\
\text { on } \\
\end{array}$ & $\begin{array}{r}.25 \\
9\end{array}$ & $\begin{array}{r}.43 \\
0\end{array}$ & $\begin{array}{r}.19 \\
1\end{array}$ & $\begin{array}{r}.04 \\
0\end{array}$ & $\begin{array}{r}.14 \\
7\end{array}$ & $\begin{array}{r}.12 \\
7\end{array}$ & $\begin{array}{r}.12 \\
7\end{array}$ & $\begin{array}{r}- \\
.13 \\
5 \\
\end{array}$ & $\begin{array}{r}.13 \\
8\end{array}$ & $\begin{array}{r}.27 \\
8\end{array}$ & $\begin{array}{r}.22 \\
3\end{array}$ & $\begin{array}{r}.33 \\
3\end{array}$ & $\begin{array}{r}- \\
.01 \\
1 \\
\end{array}$ & 1 & $\begin{array}{r}.35 \\
3\end{array}$ & $\begin{array}{r}.08 \\
3\end{array}$ \\
\hline & Sig. (2- & $\begin{array}{r}.00 \\
0\end{array}$ & $\begin{array}{r}.00 \\
0\end{array}$ & $\begin{array}{r}.00 \\
7\end{array}$ & $\begin{array}{r}.57 \\
0\end{array}$ & $\begin{array}{r}.03 \\
8\end{array}$ & $\begin{array}{r}.07 \\
3\end{array}$ & $\begin{array}{r}.07 \\
3\end{array}$ & .05 & .05 & $\begin{array}{r}.00 \\
0\end{array}$ & $\begin{array}{r}.00 \\
2\end{array}$ & $\begin{array}{r}.00 \\
0\end{array}$ & $\begin{array}{r}.87 \\
7\end{array}$ & & $\begin{array}{r}.00 \\
0\end{array}$ & $\begin{array}{r}.24 \\
3\end{array}$ \\
\hline & $\mathrm{N}$ & 200 & 200 & 200 & 200 & 200 & 200 & 200 & 200 & 200 & 200 & 200 & 200 & 200 & 200 & 200 & $\begin{array}{r}20 \\
0 \\
\end{array}$ \\
\hline \multirow{3}{*}{$\begin{array}{l}\text { Poor } \\
\text { state of } \\
\text { infrastruc } \\
\text { ture in } \\
\text { the } \\
\text { country }\end{array}$} & $\begin{array}{l}\text { Pearson } \\
\text { Correlati } \\
\text { on }\end{array}$ & $\begin{array}{r}- \\
.09 \\
7\end{array}$ & $\begin{array}{r}.35 \\
7\end{array}$ & $\begin{array}{r}.16 \\
3\end{array}$ & $\begin{array}{r}.00 \\
5\end{array}$ & $\begin{array}{r}.26 \\
7\end{array}$ & $\begin{array}{r}.38 \\
4\end{array}$ & $\begin{array}{r}.67 \\
8\end{array}$ & $\begin{array}{r}.39 \\
4\end{array}$ & $\begin{array}{r}.44 \\
6\end{array}$ & $\begin{array}{r}.41 \\
0\end{array}$ & $\begin{array}{r}.08 \\
1\end{array}$ & $\begin{array}{r}.53 \\
7\end{array}$ & $\begin{array}{r}.19 \\
1\end{array}$ & $\begin{array}{r}.35 \\
3\end{array}$ & 1 & $\begin{array}{r}- \\
.16 \\
6 \\
\end{array}$ \\
\hline & $\begin{array}{l}\text { Sig. (2- } \\
\text { tailed) }\end{array}$ & $\begin{array}{r}.17 \\
2\end{array}$ & $\begin{array}{r}.00 \\
0\end{array}$ & $\begin{array}{r}.02 \\
1\end{array}$ & $\begin{array}{r}.94 \\
0\end{array}$ & $\begin{array}{r}.00 \\
0\end{array}$ & $\begin{array}{r}.00 \\
0\end{array}$ & $\begin{array}{r}.00 \\
0\end{array}$ & $\begin{array}{r}.00 \\
0\end{array}$ & $\begin{array}{r}.00 \\
0\end{array}$ & $\begin{array}{r}.00 \\
0\end{array}$ & $\begin{array}{r}.25 \\
6\end{array}$ & $\begin{array}{r}.00 \\
0\end{array}$ & $\begin{array}{r}.00 \\
7\end{array}$ & $\begin{array}{r}.00 \\
0\end{array}$ & & $\begin{array}{r}.01 \\
9\end{array}$ \\
\hline & $\mathrm{N}$ & 200 & 200 & 200 & 200 & 200 & 200 & 200 & 200 & 200 & 200 & 200 & 200 & 200 & 200 & 200 & $\begin{array}{r}20 \\
0\end{array}$ \\
\hline \multirow{3}{*}{$\begin{array}{l}\text { Lack of } \\
\text { family } \\
\text { support }\end{array}$} & $\begin{array}{l}\text { Pearson } \\
\text { Correlati } \\
\text { on }\end{array}$ & $\begin{array}{r}.05 \\
5\end{array}$ & $\begin{array}{r}.22 \\
5\end{array}$ & $\begin{array}{r}.45 \\
6\end{array}$ & $\begin{array}{r}.43 \\
3\end{array}$ & $\begin{array}{r}- \\
.07 \\
4 \\
\end{array}$ & $\begin{array}{r}- \\
.06 \\
5 \\
\end{array}$ & $\begin{array}{r}- \\
.34 \\
6\end{array}$ & $\begin{array}{r}- \\
.07 \\
3 \\
\end{array}$ & $\begin{array}{r}.15 \\
4\end{array}$ & $\begin{array}{r}- \\
.10 \\
3 \\
\end{array}$ & $\begin{array}{r}- \\
.02 \\
5 \\
\end{array}$ & $\begin{array}{r}.03 \\
6\end{array}$ & $\begin{array}{r}- \\
.02 \\
5 \\
\end{array}$ & $\begin{array}{r}.08 \\
3\end{array}$ & $\begin{array}{r}- \\
.16 \\
6 \\
\end{array}$ & 1 \\
\hline & $\begin{array}{l}\text { Sig. (2- } \\
\text { tailed) }\end{array}$ & $\begin{array}{r}.44 \\
2\end{array}$ & $\begin{array}{r}.00 \\
1\end{array}$ & $\begin{array}{r}.00 \\
0\end{array}$ & $\begin{array}{r}.00 \\
0\end{array}$ & $\begin{array}{r}.30 \\
0 \\
\end{array}$ & $\begin{array}{r}.36 \\
1 \\
\end{array}$ & $\begin{array}{r}.00 \\
0\end{array}$ & $\begin{array}{r}.30 \\
8\end{array}$ & $\begin{array}{r}.02 \\
9\end{array}$ & $\begin{array}{r}.14 \\
6\end{array}$ & $\begin{array}{r}.72 \\
2\end{array}$ & $\begin{array}{r}.61 \\
4\end{array}$ & $\begin{array}{r}.72 \\
8\end{array}$ & $\begin{array}{r}.24 \\
3\end{array}$ & $\begin{array}{r}.01 \\
9\end{array}$ & \\
\hline & $\mathrm{N}$ & 200 & 200 & 200 & 200 & 200 & 200 & 200 & 200 & 200 & 200 & 200 & 200 & 200 & 200 & 200 & $\begin{array}{r}20 \\
0\end{array}$ \\
\hline
\end{tabular}

The KMO value of 0.594 in table 4 indicates that the interrelationships of the variables are of good precision. Bartlett's test of sphericity $(1658.206, \mathrm{df}=120)$ with an associated $p$ value of $<0.001$ also indicates that we can proceed with the research study.

Table 4: $\quad$ KMO and Bartlett's Test

\begin{tabular}{|ll|r|}
\hline Kaiser-Meyer-Olkin Measure of Sampling Adequacy. & .594 \\
& Approx. Chi-Square & 1658.206 \\
Bartlett's Test of Sphericity & df & 120 \\
& Sig. & .000 \\
\hline
\end{tabular}

The result of component analysis is shown in table 5, table 6 and figure 4. Five factors based on their variance and significance of Kaiser criterion (Kaiser,1960) were extracted among factors inhibiting respondents' decision for self-employment. These factors are difficulty in getting bank finance, inability to develop new business ideas, fear of risk, weak economic environment, and difficulty in finding customer with variance of 4.030, 2.674, 2.216, 1.194 and 1.108 respectively and of which their Eigen value is greater than one. These factors altogether explained $70.15 \%$ of the total variation. The other eleven (11) were also found to be fundamental structure of barriers but contributed only $29.85 \%$ of the total variation. 
INTERNATIONAL JOURNAL OF ACADEMIC RESEARCH IN BUSINESS AND SOCIAL SCIENCES Vol. 8, No. 12, Dec, 2018, E-ISSN: 2222-6990 @ 2018 HRMARS

Table 5

\begin{tabular}{|c|c|c|c|c|c|c|c|c|c|}
\hline \multirow[t]{2}{*}{$\begin{array}{l}\text { Compo } \\
\text { nent }\end{array}$} & \multicolumn{3}{|c|}{ Initial Eigenvalues } & \multicolumn{3}{|c|}{$\begin{array}{c}\text { Extraction Sums of Squared } \\
\text { Loadings }\end{array}$} & \multicolumn{3}{|c|}{$\begin{array}{c}\text { Rotation Sums of Squared } \\
\text { Loadings }\end{array}$} \\
\hline & Total & $\begin{array}{c}\% \text { of } \\
\text { Varianc } \\
\mathrm{e}\end{array}$ & $\begin{array}{l}\text { Cumulati } \\
\text { ve } \%\end{array}$ & Total & $\begin{array}{c}\% \text { of } \\
\text { Varianc } \\
\text { e }\end{array}$ & $\begin{array}{l}\text { Cumulativ } \\
\text { e \% }\end{array}$ & Total & $\begin{array}{c}\% \text { of } \\
\text { Varianc } \\
\mathrm{e}\end{array}$ & $\begin{array}{c}\text { Cumulativ } \\
\text { e } \%\end{array}$ \\
\hline 1 & 4.03 & 25.187 & 25.187 & 4.03 & 25.187 & 25.187 & 3.307 & 20.666 & 20.666 \\
\hline 2 & 2.674 & 16.715 & 41.902 & 2.674 & 16.715 & 41.902 & 2.62 & 16.375 & 37.041 \\
\hline 3 & 2.216 & 13.853 & 55.755 & 2.216 & 13.853 & 55.755 & 2.262 & 14.138 & 51.179 \\
\hline 4 & 1.194 & 7.462 & 63.217 & 1.194 & 7.462 & 63.217 & 1.61 & 10.06 & 61.239 \\
\hline 5 & 1.108 & 6.927 & 70.145 & 1.108 & 6.927 & 70.145 & 1.425 & 8.906 & 70.145 \\
\hline 6 & 0.916 & 5.726 & 75.871 & & & & & & \\
\hline 7 & 0.853 & 5.332 & 81.203 & & & & & & \\
\hline 8 & 0.779 & 4.866 & 86.069 & & & & & & \\
\hline 9 & 0.526 & 3.285 & 89.354 & & & & & & \\
\hline 10 & 0.42 & 2.624 & 91.978 & & & & & & \\
\hline 11 & 0.395 & 2.47 & 94.448 & & & & & & \\
\hline 12 & 0.294 & 1.836 & 96.284 & & & & & & \\
\hline 13 & 0.223 & 1.392 & 97.676 & & & & & & \\
\hline 14 & 0.164 & 1.023 & 98.699 & & & & & & \\
\hline 15 & 0.128 & 0.801 & 99.5 & & & & & & \\
\hline 16 & 0.08 & 0.5 & 100 & & & & & & \\
\hline
\end{tabular}


INTERNATIONAL JOURNAL OF ACADEMIC RESEARCH IN BUSINESS AND SOCIAL SCIENCES

Vol. 8, No. 12, Dec, 2018, E-ISSN: 2222-6990 (C) 2018 HRMARS

Table 6: Principal Component Factor Analysis (varimax rotation), Factor loadings and Communalities for the Barrier Variables

\begin{tabular}{|c|c|c|c|c|c|c|}
\hline & & & mpone & & & \\
\hline & 1 & 2 & 3 & 4 & 5 & Communalities \\
\hline Fear of Risk & 291 & .070 & .812 & . 128 & .057 & .769 \\
\hline Fear of failure & .343 & .281 & .756 & .031 & .058 & .772 \\
\hline Inability to develop new business idea & .060 & .839 & .185 & .052 & .328 & .852 \\
\hline Inability to write business plan & .095 & .806 & .027 & .112 & .346 & .791 \\
\hline Insufficient practical skill to start business & .395 & .304 & .020 & .305 & .073 & .347 \\
\hline Lack of savings & .705 & .136 & .094 & .128 & .328 & .649 \\
\hline Difficulty in obtaining bank finance & .839 & .304 & .051 & .126 & .018 & .815 \\
\hline $\begin{array}{l}\text { Complicated legal and administrative procedure for business } \\
\text { registration. }\end{array}$ & .431 & .232 &. & .458 & .206 & .644 \\
\hline Poor implementation of import related legislation & .536 & .553 & 359 & .319 & .059 & .827 \\
\hline Weak economic environment & .207 & .010 & .084 & .850 & .036 & .774 \\
\hline Fear of uncertainty & .033 & .089 & .482 & .586 & .079 & .591 \\
\hline Low demand for Nigerian made goods/services. & .660 & .192 & .250 & .011 & .345 & .654 \\
\hline Difficult to find customers & .072 & .155 & .039 & .016 & .893 & .828 \\
\hline Tough Competition & .305 & .142 & .600 & .088 & .058 & .485 \\
\hline Poor state of infrastructure in the country & .810 & .011 & .115 & .165 & .207 & .740 \\
\hline Lack of family support & .164 & .729 & .162 & 197 & .249 & .685 \\
\hline
\end{tabular}

Extraction Method: Principal Component Analysis.

Rotation Method: Varimax with Kaiser Normalization. ${ }^{\text {a }}$

a. Rotation converged in 13 iterations. 
INTERNATIONAL JOURNAL OF ACADEMIC RESEARCH IN BUSINESS AND SOCIAL SCIENCES

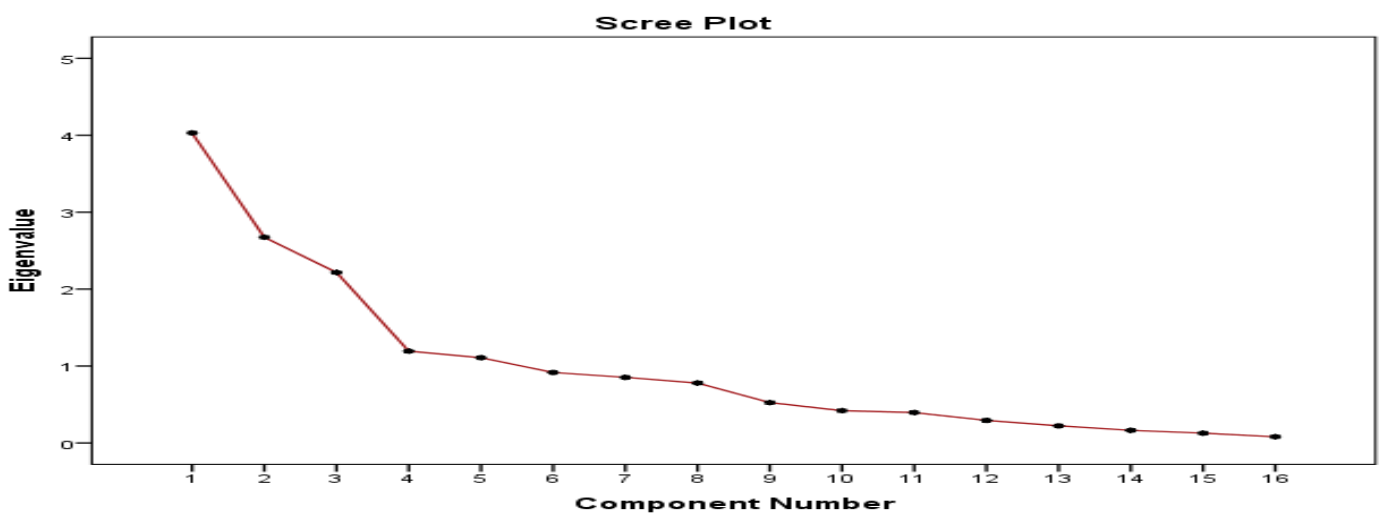

Fig: 4 Screen Plot of Ordered Eigen Value

\section{Conclusion and Recommendations}

This study empirically investigated barriers inhibiting self-employment among Nigerian graduates. Five perceived barriers were found to be significant and positively correlated with the graduates' decision to self-employment. They are difficulty in getting bank finance, inability to develop new business ideas, fear of risk, weak economic environment and difficulty in finding customers. These reported perceived barriers to business star-ups could possibly explain low engagement of Nigerian graduates into self-employment. The longer these barriers persist, the lesser would be the number of graduates engaged in self-employment. It is therefore recommended that the policy makers should with outmost priority start to implement sound economic policies that would boost the country's economic environment to create enabling environment for business start-ups; simplify the bureaucracy of accessing loan to make finance readily available for graduates entrepreneurs; and the campaign to re-orient Nigerians towards products produced in Nigeria should be intensified to widen market for locally made products. The Entrepreneurship Development Centres in various institutions of higher learning also need to do more in areas such as business advice, mentorship and practical support to make students capable of devising a way to overcome the fear of risk and develop ability to generate new business idea. Implementing the listed suggestions will go in a long way to encourage Nigerian graduates to consider self-employment as career option.

\section{References}

Abdulkarim, J. (2012). Entrepreneurship in Technical and Vocational Education. Umuahia: ljeh Concepts

Adebayo, O. \& Kolawole, J. A. (2013). The Historical Background of Entrepreneurial Development in Nigeria: its gains, shortcomings and needful. Journal of Emerging Trends in Economics and Management, 4(5), 493-500.

Carayannis, E., Evans, D. \& Hanson, M. (2003). A Cross-cultural Learning Strategy for Entrepreneurship Education: Outline of Key Concepts and Lessons Learned from a Comparative Study of Entrepreneurship Students in France and the US. Technovation, 
INTERNATIONAL JOURNAL OF ACADEMIC RESEARCH IN BUSINESS AND SOCIAL SCIENCES Vol. 8, No. 12, Dec, 2018, E-ISSN: 2222-6990 @ 2018 HRMARS

23(9), 757-771.

Ekpoh, U. I. \& Edet, A. O. (2011). Entrepreneurship Education and Career Intentions of Tertiary Education Students in Akwa Ibom and Cross Rivers States, Nigeria. International Education Studies, 4(1), 172-178.

Harrison, R. \& Hart, M. (1989). Factors Influencing New Business Formation: A Case Study Of Northern Ireland. Environment and Planning 15(10).

lakovleva, T. A, Kolvereid, L., Gorgievski, M. J., \& Sorhaug, O. (2014). Comparison of Perceived Barriers to Entrepreneurship in Eastern and Western European Countries. International Journal of Entrepreneurship and Innovation Management, 18(2/3): 115133.

Koleveried, L. \& Isaksen, E. (2006). New business start-up and subsequent entry into Self-employment. Journal of Business Venturing, 15(5), 411-432.

Luthje, C. \& Franke, N. (2003). The 'making' of an entrepreneur: Testing a Model of Entrepreneurial intent among engineering students at MIT. Research and Development Management, 33(2), 135-147.

Mauchi, F. N., Karambakuwa, R. T., Gopo, R. N., Kosmas, N., Mangwende, S. \& Gombarume, F. B. (2011). Entrepreneurship Education Lessons: A Case of Zimbabwe Tertiary Education Institutions. International Research Journals, 2(7), 1306-1311.

Nnadozie, C.O., Akanwa, P. C. \& Nnadozie, C. D. (2013). Impact of Entrepreneurship Education on the Career Aspirations of Nigerian Para-Professional Librarians-inTraining. Academic Journal of Interdisciplinary Studies, 2(5), 149-161.

Onuma, N. (2016). Entrepreneurship Education in Nigerian Tertiary Institutions: A Remedy To Graduates Unemployment. British Journal of Education, 4(5), 16-28.

Owusu-Ansah, W. \& Poku, K. (2012). Entrepreneurship Education, A Panacea to Graduate Unemployment in Ghana. International Journal of Humanities and Social Science, 2(15), 211-220.

Pittaway, L. \& Cope, J. (2007). Entrepreneurship Education: A Systematic Review of the Evidence. International Small Business Journal, 25(5), 479-510.

Rosni, B., Md Aminul I. \& Jocelyne, L. (2015). Entrepreneurship Education: Experiences in Selected Countries. International Education Studies, 8(1), 88-99.

Sandhu, M. S, Sidique, S. F, \& Riaz, S. (2011). Entrepreneurship barriers and Entrepreneurial inclination among Malaysian postgraduate students. International Journal of Entrepreneurial Behaviour and Research, 17(4), 428-449.

Sitaridis, I. K. \& Kitsios, F. (2016). Students' Perceptions of Barriers to Entrepreneurship

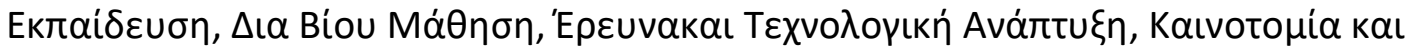
Оıкоvонi $\alpha, 1:$ 524-535.

Smith, K. \& Beasley, M. (2011). Graduate Entrepreneurs: Intentions, Barriers and Solutions. Journal of Education and Training, 54(8), 722-740.

Tanveer, M. A., Gillani, U. A., Rizvi, S., Latif, M., Maqbool, H. \& Rizwan, M. (n.d). Barriers For Business Studetns in becoming an Entrpreneur in Pakistan. Journal of Business and Management, 1, 74-82. Retrieved from www.iosrjournals.org/iosr-jbm/papers/ndbmrvolume-1/J.pdf 
INTERNATIONAL JOURNAL OF ACADEMIC RESEARCH IN BUSINESS AND SOCIAL SCIENCES Vol. 8, No. 12, Dec, 2018, E-ISSN: 2222-6990 @ 2018 HRMARS

Towobola, W. L. \& Raimi, L. (2011). Open Distance Learning (ODL): A Catalyst for Educational and Entrepreneurship Development in Nigeria. Continent Journal of Educational Research, 4(3), 1-11

Van der Zwan, P., Zuurhout, P. \& Hessels, J. (2013). Entrepreneurship Education and SelfEmployment: The Role of Perceived Barriers. EIM Research Report, Retrieved from www. Entrepreneurship-sme.eu. 